\title{
Circulation Journal Awards for the Year 2019
}

\author{
Toyoaki Murohara, MD, $\mathrm{PhD}$
}

\section{Dear Colleagues,}

On behalf of the Editorial Team of Circulation Journal, I am pleased to announce the Circulation Journal Awards for the Year 2019.

The aim of these Awards is to recognize papers published in 2019, both clinical and experimental studies, that were highly appreciated by the Editorial Team. The selection process comprises 2 steps. In the first step, from 248 original papers published in the Journal in 2019, our 42 Japanese Associate Editors selected papers with a high scientific level in their respective fields, and in the second step, the 4 Associate Editorial Teams (10 11 on 1 team) further evaluated the selected papers in terms of originality, contribution to cardiovascular science, manner of paper preparation, and future possibilities.

In the year of 2019, the following 7 papers have been selected for the Circulation Journal Awards.

First Place in the Clinical Investigation Section

(Circ J 2019; 83: 831-836)1

\section{Polysplenia Syndrome as a Risk Factor for Early Progression of Pulmonary Hypertension \\ Akimichi Shibata, Hiroki Mori, Kazuki Kodo, Toshio Nakanishi, Hiroyuki Yamagishi \\ (Division of Pediatric Cardiology, Department of Pediatrics, Keio University School of Medicine, Tokyo (A.S., K.K., H.Y.); Department of Pediatric Cardiology, Tokyo Women's Medical University, Tokyo (H.M., T.N.), Japan)}

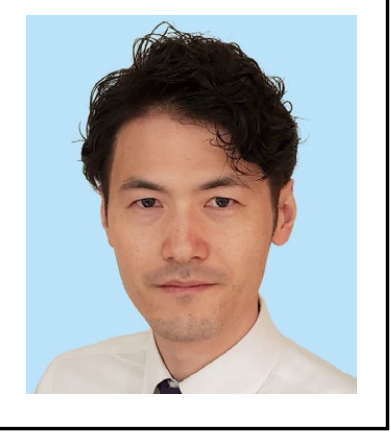

Background: Recent progress in surgical and intensive care has improved the prognosis of congenital heart disease (CHD) associated with heterotaxy syndrome. Less is known, however, about pulmonary vascular complications in these patients.

Methods and Results: We reviewed medical records of 236 patients who were diagnosed with polysplenia syndrome at 2 institutions for pediatric cardiology in Japan from 1978 to 2015. We selected and compared the clinical records of 16 patients with polysplenia who had incomplete atrioventricular septal defect (AVSD) as the polysplenia group, and 22 age-matched patients with incomplete AVSD without any syndromes including polysplenia as the control group. Although the severity of systemic to pulmonary shunt was not significantly different between the groups, mean pulmonary artery pressure (mPAP) and pulmonary vascular resistance index (PVRI) were significantly higher in the polysplenia group than the control (mPAP, 37.3 vs. $19.1 \mathrm{mmHg}, \mathrm{P}=0.001$; PVRI, $5.7 \mathrm{vs.} 1.4 \mathrm{WU} \cdot \mathrm{m}^{2}, \mathrm{P}=0.014$ ) before surgical intervention. On regression analysis, polysplenia influenced the development of pulmonary hypertension $(\mathrm{PH})$ regardless of age at evaluation or degree of systemic to pulmonary shunt in the patients with incomplete AVSD.

Conclusions: Polysplenia syndrome is an independent risk factor for CHD-associated PH. Earlier intervention may be required to adjust the pulmonary blood flow in polysplenia syndrome with CHD to avoid the progression of $\mathrm{PH}$.

J-STAGE Advance Publication released online February 14, 2020

Department of Cardiology, Nagoya University Graduate School of Medicine, Nagoya, Japan

Mailing address: Toyoaki Murohara, MD, PhD, Department of Cardiology, Nagoya University Graduate School of Medicine, 65

Tsurumai, Showa-ku, Nagoya 466-8550, Japan. E-mail: murohara@med.nagoya-u.ac.jp

ISSN-1346-9843 All rights are reserved to the Japanese Circulation Society. For permissions, please e-mail: cj@j-circ.or.jp 


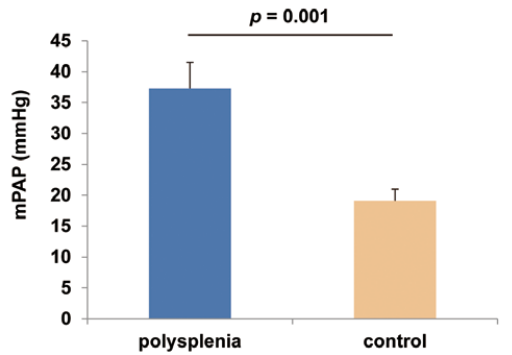

B

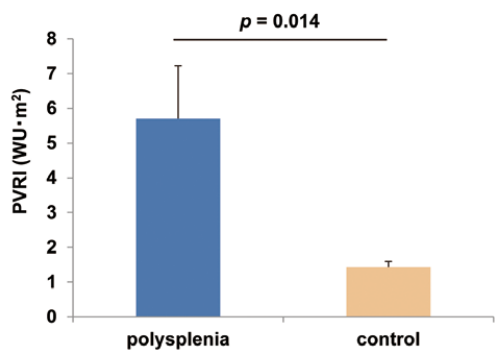

Figure 1. (A) Mean pulmonary artery pressure (mPAP) and (B) pulmonary vascular resistance index (PVRI) according to presence of polysplenia in patients with incomplete atrioventricular septal defect. Data given as mean and standard error.
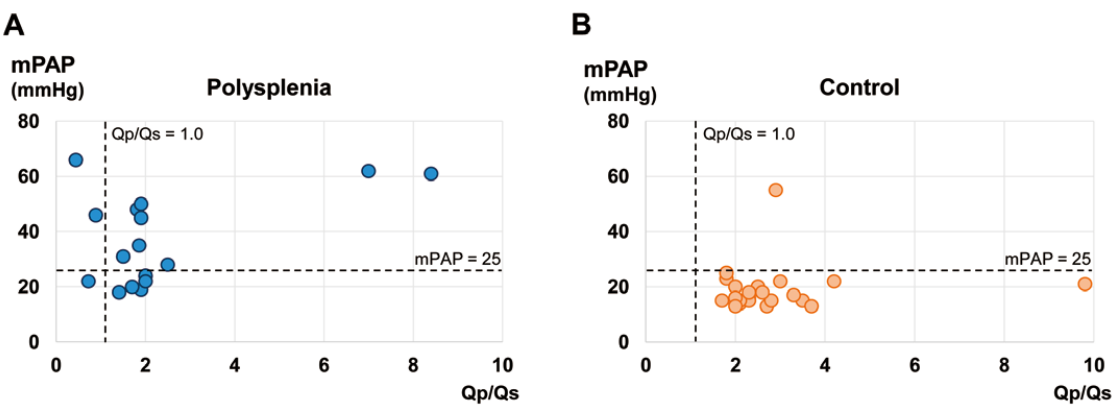

Figure 2. Mean pulmonary artery pressure (mPAP) vs. ratio of pulmonary to systemic blood flow (Qp/Qs) in the (A) polysplenia group (polysplenia+incomplete atrioventricular septal defect [AVSD]; $n=16$ ); and (B) control group (incomplete AVSD; $n=22$ ).

\section{Efficacy and Safety of Ivabradine in Japanese Patients With Chronic Heart} Failure - J-SHIFT Study -

Hiroyuki Tsutsui, Shin-ichi Momomura, Akira Yamashina, Hiroaki Shimokawa, Yasuki Kihara, Yoshihiko Saito, Nobuhisa Hagiwara, Hiroshi Ito, Masafumi Yano, Kazuhiro Yamamoto, Junya Ako, Takayuki Inomata, Yasushi Sakata, Takashi Tanaka, Yasushi Kawasaki on behalf of the J-SHIFT Study Investigators (Department of Cardiovascular Medicine, Graduate School of Medical Sciences, Kyushu University, Fukuoka (H.T.); Cardiovascular Division, Jichi Medical University, Saitama Medical Center, Saitama (S.M.); Department of Cardiology, Tokyo Medical University, Tokyo (A. Y.); Department of Cardiovascular Medicine, Tohoku University Graduate School

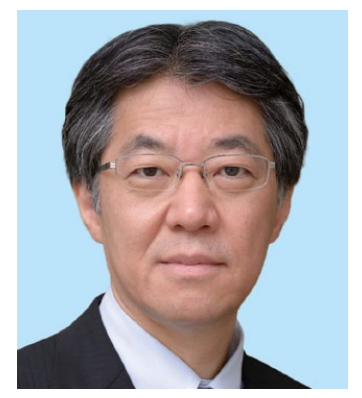
of Medicine, Sendai (H.S. ); Department of Cardiovascular Medicine, Hiroshima University Graduate School of Biomedical \& Health Sciences, Hiroshima (Y. Kihara); First Department of Internal Medicine, Nara Medical University, Kashihara (Y. Saito); Department of Cardiology, Tokyo Women's Medical University, Tokyo (N.H.); Department of Cardiovascular Medicine, Okayama University Graduate School of Medicine, Dentistry and Pharmaceutical Sciences, Okayama (H.I.); Division of Cardiology, Department of Medicine and Clinical Science, Yamaguchi University Graduate School of Medicine, Ube (M.Y.); Division of Cardiovascular Medicine, Endocrinology and Metabolism, Department of Molecular Medicine and Therapeutics, Faculty of Medicine, Tottori University, Yonago (K. Y.); Cardiovascular Medicine, Kitasato University, Sagamihara (J.A.); Cardiovascular Medicine, Kitasato University Kitasato Institute Hospital, Tokyo (T.I.); Department of Cardiovascular Medicine, Graduate School of Medicine, Osaka University, Suita (Y. Sakata); and Department of Clinical Development, Ono Pharmaceutical Co., Ltd., Osaka (T.T., Y. Kawasaki), Japan) 


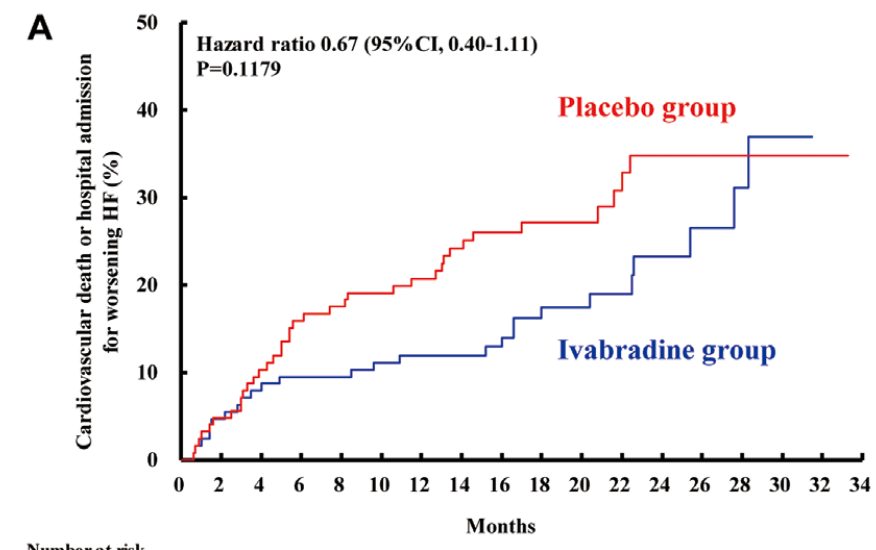

Number at risk

$\begin{array}{lllllllllllllllllll}\text { Ivabradine group } & 127 & 120 & 116 & 114 & 114 & 112 & 109 & 95 & 80 & 68 & 55 & 42 & 31 & 20 & 13 & 5 & 0 & 0\end{array}$ $\begin{array}{lllllllllllllllllll}\text { Placebo group } & 127 & 120 & 113 & 105 & 103 & 101 & 97 & 84 & 68 & 53 & 45 & 34 & 25 & 20 & 15 & 8 & 2 & 0\end{array}$

B

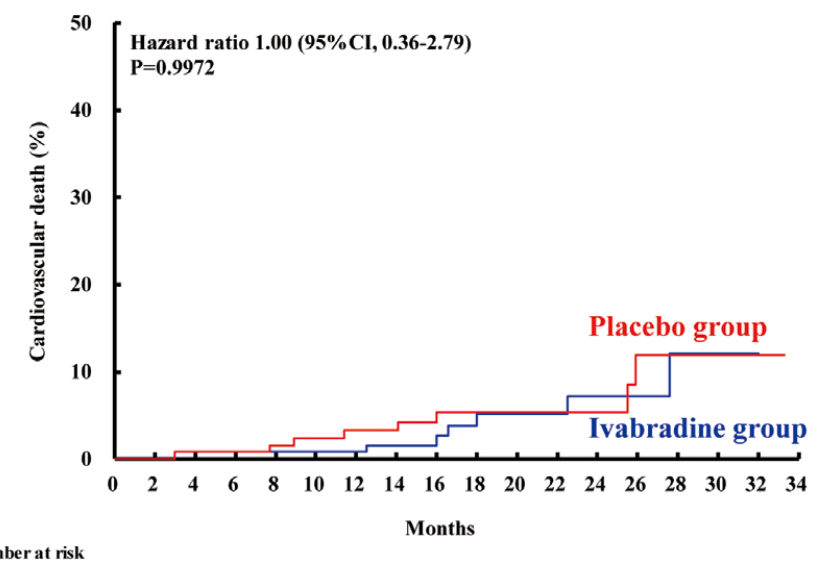

$\begin{array}{llllllllllllllllllll}\text { Ivabradine group } & 127 & 126 & 124 & 123 & 123 & 122 & 120 & 105 & 91 & 74 & 60 & 48 & 38 & 25 & 16 & 7 & 0 & 0\end{array}$ $\begin{array}{lllllllllllllllllll}\text { Placebo group } & 127 & 126 & 124 & 122 & 120 & 119 & 114 & 105 & 88 & 72 & 61 & 47 & 37 & 26 & 18 & 9 & 2 & 0\end{array}$

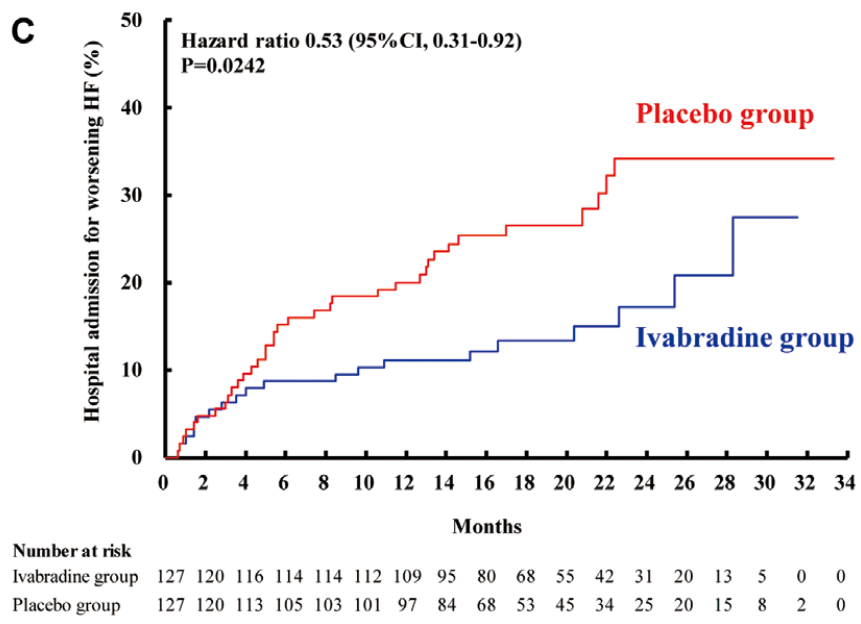

Figure 3. Kaplan-Meier cumulative event curves for $(\mathbf{A})$ the primary composite endpoint of cardiovascular death or hospital admission for worsening heart failure, (B) cardiovascular death, and (C) hospital admission for worsening heart failure. Censoring data are not displayed. $\mathrm{Cl}$, confidence interval.

Background: Increased heart rate (HR) is an independent risk factor for cardiovascular outcomes in chronic heart failure (HF). Ivabradine, an If inhibitor, improved outcomes in patients with HF and reduced ejection fraction (HFrEF) in the SHIFT study. We evaluated its efficacy and safety in Japanese HFrEF patients in a randomized, double-blind, placebo-controlled phase III study: the J-SHIFT study. The main objective was to confirm a hazard ratio of $<1$ in the primary composite endpoint of cardiovascular death or hospital admission for worsening $\mathrm{HF}$. 
Methods and Results: Patients with NYHA functional class II-IV, left ventricular EF $\leq 35 \%$, and resting HR $\geq 75$ beats/min in sinus rhythm under optimal medical therapy received ivabradine $(n=127)$ or placebo $(n=127)$. Mean reduction in resting HR was significantly greater in the ivabradine group ( $15.2 \mathrm{vs.} 6.1$ beats $/ \mathrm{min}, \mathrm{P}<0.0001)$. However, symptomatic bradycardia did not occur. A total of $26(20.5 \%)$ patients in the ivabradine group and 37 (29.1\%) patients in the placebo group had the primary endpoint event (hazard ratio $0.67,95 \%$ CI $0.40-1.11$, $\mathrm{P}=0.1179)$ during median follow-up of 589 days. Mild phosphenes were reported in $8(6.3 \%)$ patients in the ivabradine group and $4(3.1 \%)$ patients in the placebo group $(\mathrm{P}=0.3760)$.

Conclusions: The J-SHIFT study supported the efficacy and safety of ivabradine for Japanese HFrEF patients, in accord with the SHIFT study.

Second Place in the Clinical Investigation Section

(Circ J 2019; 83: 130-138)3

\section{Cardiac Function and Type of Mitral Valve Surgery Affect Postoperative Blood Flow Pattern in the Left Ventricle}

Shohei Yoshida, Shigeru Miyagawa, Satsuki Fukushima, Yasushi Yoshikawa, Hiroki Hata, Shunsuke Saito, Daisuke Yoshioka, Satoshi Kainuma, Keitaro Domae, Ryohei Matsuura, Satoshi Nakatani, Koichi Toda, Yoshiki Sawa

(Department of Cardiovascular Surgery (S.Y., S.M., S.F., Y.Y., H.H., S.S., D. Y., S.K., K.D., R.M., K.T., Y.S. ), Department of Cardiovascular Medicine (S.N. ), Osaka University Graduate School of Medicine, Suita, Japan)
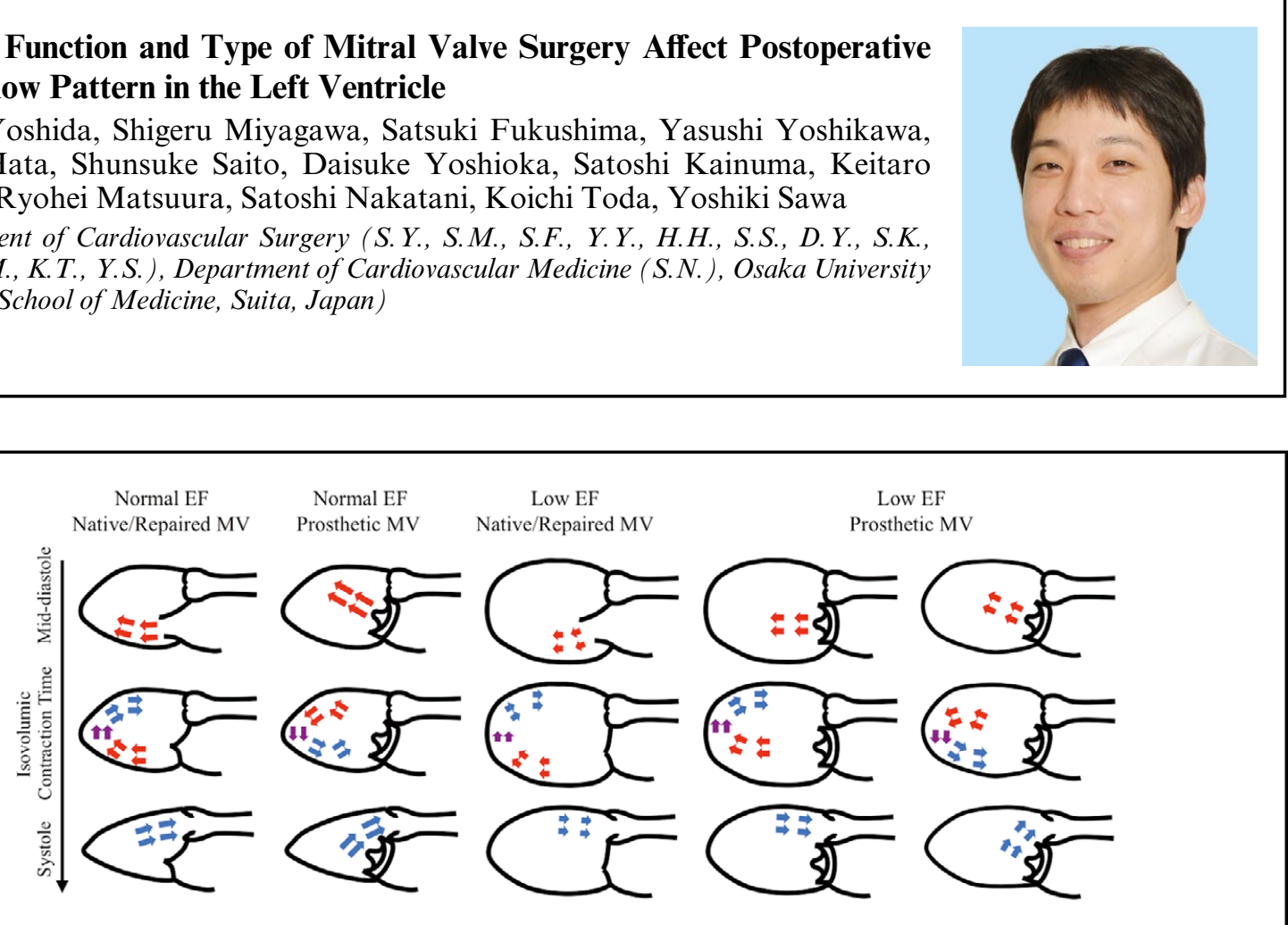

Figure 6. Schema of left ventricular vortical blood flow in all groups. (Upper) Mid-diastolic phase. (Middle) Isovolumic contraction time. (Lower) Systolic phase. EF, ejection fraction; MV, mitral valve.

Background: To determine the impact of cardiac function and type of mitral valve (MV) surgery on blood flow and energy loss in the left ventricle (LV).

Methods and Results: This study enrolled patients with ejection fraction (EF) $<35 \%$ or $>50 \%$; both groups had native $(n=27$ and $n=16)$, repaired $(n=19$ and $n=33)$, or prosthetic MVs $(n=18$ and $n=19)$. They were examined by echocardiography-based vector flow mapping to assess the LV blood flow pattern and energy loss per heartbeat. Among patients with preserved EF, those with native MVs displayed a clockwise vortex and relatively low energy loss. In contrast, MV replacement induced a counterclockwise vortex producing higher energy loss than MV repair, which induced a normal clockwise vortex. This indicated the need for MV repair to minimize LV energy loss after surgery. Among the patients with reduced EF, those with native MVs showed a blood flow pattern similar to those with preserved EF and native MVs; furthermore, those with repaired MVs and half of the patients with prosthetic MVs displayed a clockwise vortex, resulting in no difference in energy loss between the 2 types of MV surgery.

Conclusions: Cardiac function and the type of MV surgery are factors affecting the postoperative LV blood flow pattern. MV replacement resulted in abnormal blood flow with normal cardiac function, whereas advanced cardiomyopathy modified the blood flow pattern post-MV replacement. 


\section{Cardiovascular Risk Assessment Chart by Dietary Factors in Japan - NIPPON DATA80 -}

Keiko Kondo, Katsuyuki Miura, Sachiko Tanaka-Mizuno, Aya Kadota, Hisatomi Arima, Nagako Okuda, Akira Fujiyoshi, Naoko Miyagawa, Katsushi Yoshita, Tomonori Okamura, Akira Okayama, Hirotsugu Ueshima for the NIPPON DATA80 Research Group

(Department of Public Health (K.K., K.M., A.K., A.F., N.M., H.U.), Center for Epidemiologic Research in Asia (K.M., A.K., H.U.), Department of Medical Statistics (S.T.-M.), Shiga University of Medical Science, Shiga; Department of Preventive Medicine and Public Health, Faculty of Medicine, Fukuoka University, Fukuoka (H.A.); Department

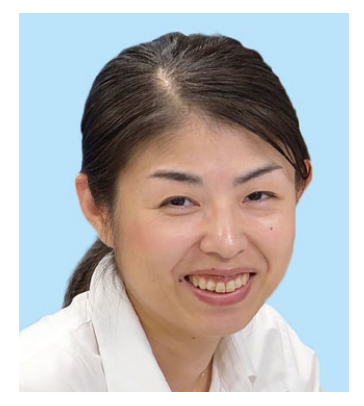
of Health and Nutrition, University of Human Arts and Sciences, Saitama (N.O.);

Department of Hygiene, Wakayama Medical University, Wakayama (A.F.); International Center for Nutrition and Information, National Institute of Health and Nutrition, National Institutes of Biomedical Innovation, Health and Nutrition, Tokyo (N.M.); Department of Food and Nutrition, Osaka City University, Osaka (K. Y.); Department of Preventive Medicine and Public Health, Keio University School of Medicine, Tokyo (T.O.); and Research Institute of Strategy for Prevention, Tokyo (A.O.), Japan)

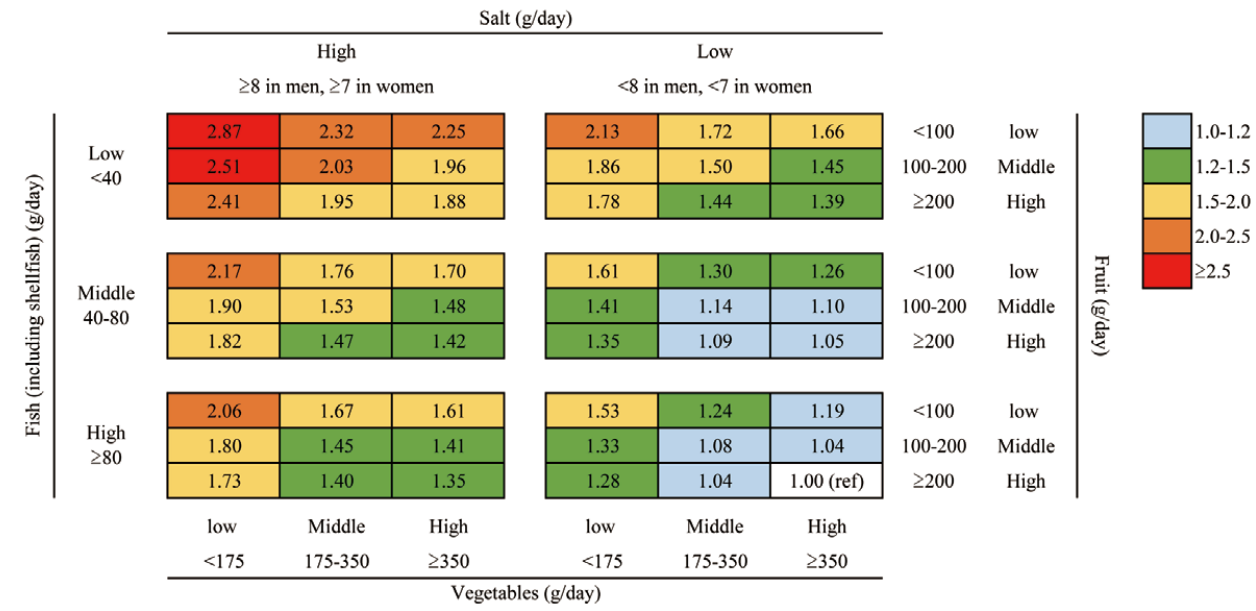

Figure. Risk assessment chart for death from cardiovascular diseases according to intake of different dietary factors, calculated using data from 4,002 men and 5,113 women aged 30-79 years from the NIPPON DATA80 study. The hazard ratio (HR) of cardiovascular disease mortality according to the dietary intake of a combination of factors (vegetables, fruit, fish, and salt) was calculated by multiplying together the HRs for each of the dietary factors.

Background: Many studies show that dietary factors such as vegetables, fruit, and salt are associated with cardiovascular disease (CVD) risk. However, a risk assessment chart for CVD mortality according to combinations of dietary factors has not been established.

Methods and Results: Participants were 9,115 men and women aged 30-79 years enrolled in the National Nutritional Survey of Japan in 1980 with a 29-year follow-up. Dietary intake was assessed using a 3-day weighed dietary record at baseline. Cox regression models were used to estimate the hazard ratio (HR) of CVD mortality stratified by vegetables, fruit, fish, and salt consumption. HRs of CVD mortality according to combinations of dietary factors were color coded on an assessment chart. Higher intakes of vegetables, fruit, and fish, and lower salt intake were associated with lower CVD mortality risk. HRs calculated from combinations of dietary factors were displayed using 5 colors corresponding to the magnitude of the HR. People with the lowest intake of vegetables, fruit, and fish, and higher salt intake had a HR of 2.87 compared with those with the highest intake of vegetables, fruit, and fish, and lower salt intake.

Conclusions: Vegetables, fruit, fish, and salt intake were independently associated with CVD mortality risk. The assessment chart generated could be used in Japan as an educational tool for CVD prevention. 


\section{Working Hours and Risk of Acute Myocardial Infarction and Stroke Among Middle-Aged Japanese Men - The Japan Public Health Center-Based Prospective Study Cohort II -}

Rie Hayashi, Hiroyasu Iso, Kazumasa Yamagishi, Hiroshi Yatsuya, Isao Saito, Yoshihiro Kokubo, Ehab S. Eshak, Norie Sawada, Shoichiro Tsugane for the Japan Public Health Center-Based (JPHC) Prospective Study Group

(Public Health, Department of Social Medicine, Osaka University Graduate School of Medicine, Suita (R.H., H.I., E.S.E.); Department of Public Health Medicine, Faculty of Medicine, University of Tsukuba, Tsukuba (H.I., K. Y.); Department of Public Health, Fujita Health University, School of Medicine, Toyoake (H.Y.); Department of Community Health Systems Nursing, Ehime University Graduate School of Medicine, Toon (I.S. ); Department of Preventive Cardiology, National Cerebral and Cardiovascular Center, Suita (Y.K.), Japan; Department of Public Health and Preventive Medicine, Faculty of Medicine Minia University, Minya (E.S.E.), Egypt; and Epidemiology and Prevention Group, Center for Public Health Sciences, National Cancer Center, Tokyo (N.S., S.T.), Japan)

\begin{tabular}{|c|c|c|c|c|c|}
\hline \multirow[b]{3}{*}{ Salaried employees } & \multicolumn{4}{|c|}{ Working hours (h/day) } & \multirow{2}{*}{ P for trend } \\
\hline & \multirow[t]{2}{*}{$<7$} & \multirow[t]{2}{*}{7 to $<9$} & \multirow[t]{2}{*}{9 to $<11$} & \multirow[t]{2}{*}{$\geq 11$} & \\
\hline & & & & & \\
\hline Person-years & 4,018 & 52,059 & 37,700 & 12,070 & \\
\hline No. at risk at baseline & 129 & 3,944 & 3,196 & 858 & \\
\hline No. at risk at 10 -year survey & 306 & 1,743 & 881 & 464 & \\
\hline No. of cases & 8 & 33 & 27 & 10 & \\
\hline Multivariable-adjusted $\mathrm{HR}(95 \% \mathrm{Cl})^{\dagger}$ & $2.49(1.12-5.56)$ & 1.00 & $1.46(0.87-2.46)$ & $2.11(1.03-4.35)$ & 0.08 \\
\hline \multicolumn{6}{|l|}{ Non-salaried employees } \\
\hline Person-years & 27,895 & 47,037 & 35,122 & 15,062 & \\
\hline No. at risk at baseline & 552 & 2,586 & 2,706 & 1,173 & \\
\hline No. at risk at 10 -year survey & 2,483 & 2,449 & 1,020 & 430 & \\
\hline No. of cases & 29 & 34 & 25 & 14 & \\
\hline Multivariable-adjusted $\mathrm{HR}(95 \% \mathrm{Cl})^{\dagger}$ & $1.25(0.73-2.13)$ & 1.00 & $1.11(0.65-1.90)$ & $1.48(0.78-2.81)$ & 0.31 \\
\hline \multicolumn{6}{|l|}{ Age, 40-49 years } \\
\hline Person-years & 13,720 & 63,116 & 48,632 & 19,573 & \\
\hline No. at risk at baseline & 260 & 3,580 & 3,386 & 1,226 & \\
\hline No. at risk at 10 -year survey & 1,212 & 3,235 & 1,807 & 878 & \\
\hline No. of cases & 10 & 30 & 28 & 10 & \\
\hline Multivariable-adjusted $\mathrm{HR}(95 \% \mathrm{Cl})^{\ddagger}$ & $1.03(0.45-2.34)$ & 1.00 & $1.24(0.71-2.15)$ & $0.94(0.42-2.10)$ & 0.87 \\
\hline \multicolumn{6}{|l|}{ Age, $50-59$ years } \\
\hline Person-years & 29,740 & 43,387 & 29,625 & 9,435 & \\
\hline No. at risk at baseline & 434 & 3,016 & 2,559 & 816 & \\
\hline No. at risk at 10 -year survey & 2,883 & 1,759 & 677 & 221 & \\
\hline No. of cases & 40 & 46 & 30 & 18 & \\
\hline Multivariable-adjusted $\mathrm{HR}(95 \% \mathrm{Cl})^{\ddagger}$ & $1.52(0.85-2.71)$ & 1.00 & $1.25(0.75-2.07)$ & $2.60(1.42-4.77)$ & 0.004 \\
\hline
\end{tabular}

$\mathrm{HR}$, hazard ratio; $\mathrm{Cl}$, confidence interval. ${ }^{*}$ Calculated among $\geq 7 \mathrm{~h} /$ day workers. ${ }^{\dagger}$ Adjusted for age, body mass index, history of hypertension, history of diabetes mellitus, history of hyperlipidemia, smoking, alcohol consumption, hours of walking or standing, and sleep duration

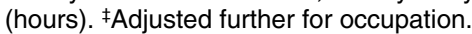

Background: Evidence from prospective cohort studies regarding the relationship between working hours and risk of cardiovascular disease is limited.

Methods and Results: The Japan Public Health Center-Based Prospective Study Cohort II involved 15,277 men aged 40-59 years at the baseline survey in 1993. Respondents were followed up until 2012. During the median 20 years of follow up (257,229 person-years), we observed 212 cases of acute myocardial infarction and 745 stroke events. Cox proportional hazards models adjusted for sociodemographic factors, cardiovascular risk factors, and 
occupation showed that multivariable-adjusted hazard ratios (HRs) associated with overtime work of $\geq 11 \mathrm{~h} /$ day were: $1.63(95 \%$ confidence interval [CI] 1.01-2.63) for acute myocardial infarction and $0.83(95 \% \mathrm{CI} 0.60-1.13)$ for total stroke, as compared with the reference group (working 7 to $<9 \mathrm{~h} /$ day). In the multivariable model, increased risk of acute myocardial infarction associated with overtime work of $\geq 11 \mathrm{~h} /$ day was more evident among salaried employees (HR 2.11, 95\% CI 1.03-4.35) and men aged 50-59 years (HR 2.60, 95\% CI 1.42-4.77). Conclusions: Among middle-aged Japanese men, working overtime is associated with a higher risk of acute myocardial infarction.

Second Place in the Experimental Investigation Section

(Circ J 2019; 83: 368-378)6

\section{Loss of Endogenous HMGB2 Promotes Cardiac Dysfunction and Pressure Overload-Induced Heart Failure in Mice}

Michio Sato, Keishi Miyata, Zhe Tian, Tsuyoshi Kadomatsu, Yoshihiro Ujihara, Jun Morinaga, Haruki Horiguchi, Motoyoshi Endo, Jiabin Zhao, Shunshun Zhu, Taichi Sugizaki, Kimihiro Igata, Masashi Muramatsu, Takashi Minami, Takashi Ito, Marco E Bianchi, Satoshi Mohri, Kimi Araki, Koichi Node, Yuichi Oike

(Department of Molecular Genetics (M.S., K.M., Z.T., T.K., J.M., H.H., M.E., J.Z., S.Z., T.S., K.I., Y.O.), Department of Immunology, Allergy and Vascular Medicine (K.M.), Center for Metabolic Regulation of Healthy Aging (K.A., Y.O. ), Graduate School of Medical Sciences, Kumamoto University, Kumamoto; Department of Cardiovascular Medicine, Saga University, Saga (M.S., K.N.); First Department of Physiology, Kawasaki Medical School, Okayama (Y.U., S.M.); Division of Molecular and Vascular Biology (M.M., T.M.), Division of Developmental Genetics (K.A.), Institute of Resource Development and Analysis, Kumamoto University, Kumamoto; Department of Systems Biology in Thromboregulation, Kagoshima University Graduate School of Medical and Dental Science, Kagoshima (T.I.), Japan; and Chromatin Dynamics Unit, San Raffaele University and Scientific Institute, Milan (M.E. B. ), Italy)

Background: The rapid increase in the number of heart failure (HF) patients in parallel with the increase in the number of older people is receiving attention worldwide. HF not only increases mortality but decreases quality of life, creating medical and social problems. Thus, it is necessary to define molecular mechanisms underlying HF development and progression. HMGB2 is a member of the high-mobility group superfamily characterized as nuclear proteins that bind DNA to stabilize nucleosomes and promote transcription. A recent in vitro study revealed that HMGB2 loss in cardiomyocytes causes hypertrophy and increases HF-associated gene expression. However, it's in vivo function in the heart has not been assessed.

Methods and Results: Western blotting analysis revealed increased HMGB2 expression in heart tissues undergoing pressure overload by transverse aorta constriction (TAC) in mice. Hmgb2 homozygous knockout (Hmgb2 ${ }^{-/-}$) mice showed cardiac dysfunction due to AKT inactivation and decreased sarco(endo)plasmic reticulum $\mathrm{Ca}^{2+}$-ATPase (SERCA)2a activity. Compared to wild-type mice, $\mathrm{Hmgb2^{-/ }}$ mice had worsened cardiac dysfunction after TAC surgery, predisposing mice to HF development and progression.

Conclusions: This study demonstrates that upregulation of cardiac HMGB2 is an adaptive response to cardiac stress, and that loss of this response could accelerate cardiac dysfunction, suggesting that HMGB2 plays a cardioprotective role. 
A

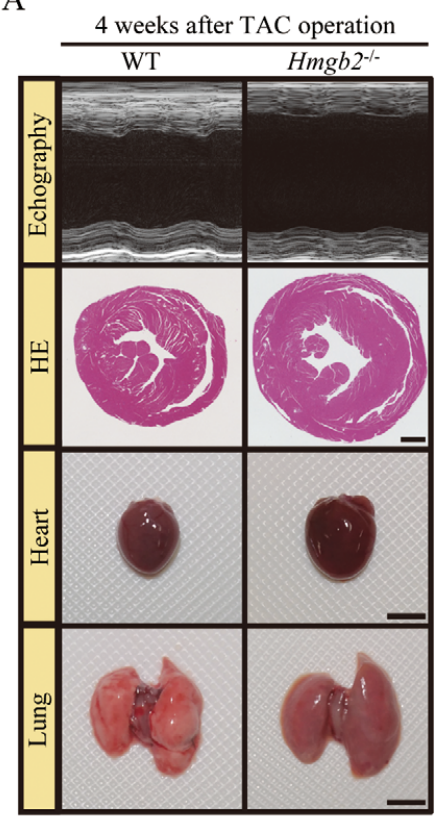

D

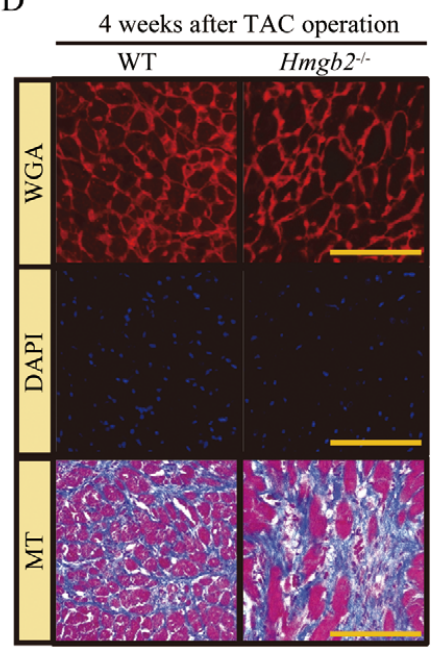

B
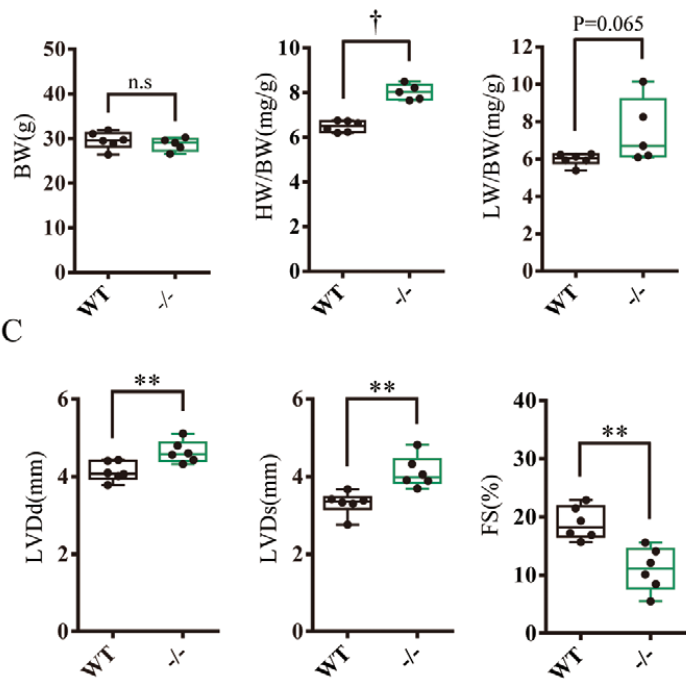

E
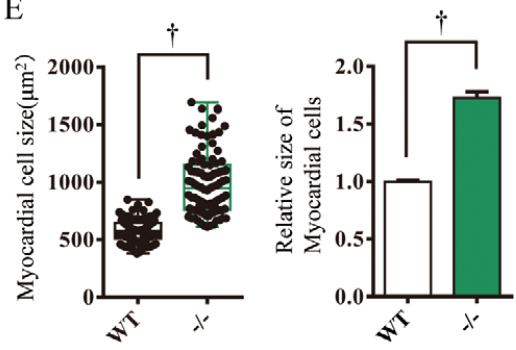

$\mathrm{F}$

G
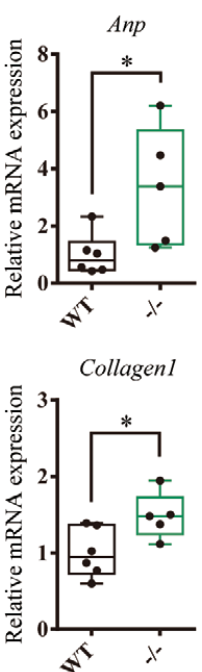
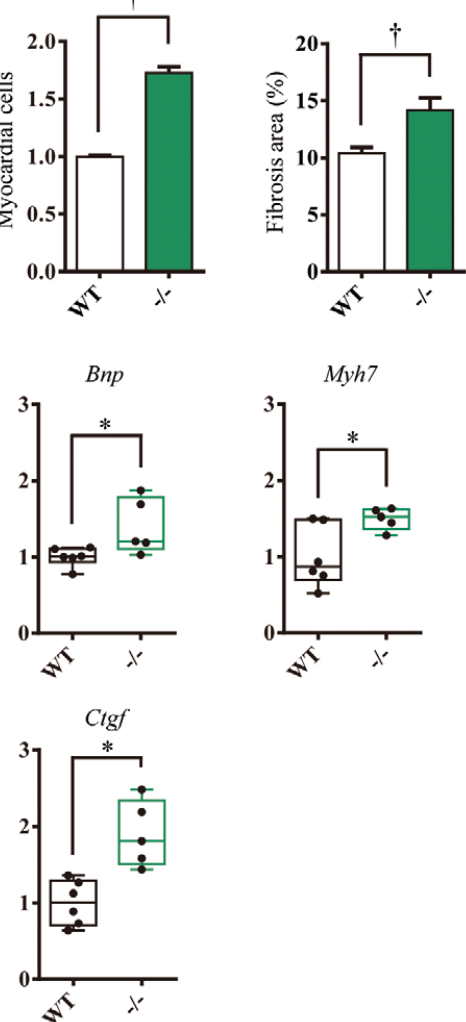

Figure 4. Hmgb2-1- mice show enhanced development of transverse aorta constriction (TAC)-induced heart failure. (A) Shown are representative M-mode echocardiography recordings (top row), hematoxylin-eosin (HE)-stained sections of heart mid-portion (second row, Scale bar, $1 \mathrm{~mm}$ ), gross appearance of a whole heart (third row, Scale bar, $5 \mathrm{~mm}$ ) and lung (fourth row, Scale bar, $5 \mathrm{~mm}$ ) and sections of Masson's Trichrome (MT)-stained heart tissue (bottom row, Scale bar, $100 \mu \mathrm{m}$ ) from 12-week-old Hmgb2-- (-/-) and wild-type (WT) littermate controls 4 weeks after TAC surgery (n=5-6 per group). (B) BW (g), heart weight per body weight (HW/BW) ratio $(\mathrm{mg} / \mathrm{g})$ and lung weight per body weight $(\mathrm{LW} / \mathrm{BW}) \mathrm{ratio}(\mathrm{mg} / \mathrm{g})$. (C) Left ventricular end-diastolic diameter (LVDd), left ventricular end-systolic diameter (LVDs) and percent fractional shortening (\%FS). (D) Shown are representative left ventricle sections stained with wheat germ agglutinin (WGA; as an indicator of cardiomyocyte size) (Upper, Scale bar, $100 \mu \mathrm{m}$ ) and 4',6-diamidino-2-phenylindole (DAPI) (Lower, Scale bar, $100 \mu \mathrm{m})$. (E) Distribution of myocardial cell size ( $\mu \mathrm{m}^{2}$, Left) and changes in relative cardiomyocyte size (Right). (F) Percentage of fibrosis area (\%). (G) Relative expression of genes associated with heart failure and fibrosis in hearts of 12 -week-old $\mathrm{Hmgb2}^{-1-}$ mice relative to littermate mice. WT values were set to $1(n=5-6$ per group). Data are presented as means \pm SEM. Statistical significance was determined by using an unpaired Student's t-test. ${ }^{\star} P<0.05,{ }^{* *} P<0.01,{ }^{~} P<0.001$ between groups. n.s., not significant. 


\section{Prognostic Impact of $\boldsymbol{\beta}$-Blocker Dose After Acute Myocardial Infarction}

Doyeon Hwang, Joo Myung Lee, Hyun Kuk Kim, Ki Hong Choi, Tae-Min Rhee, Jonghanne Park, Taek Kyu Park, Jeong Hoon Yang, Young Bin Song, Jin-Ho Choi, Joo-Yong Hahn, Seung-Hyuk Choi, Bon-Kwon Koo, Young Jo Kim, Shung-Chull Chae, Myeong Chan Cho, Chong Jin Kim, Hyeon-Cheol Gwon, Myung Ho Jeong, Hyo-Soo Kim, The KAMIR Investigators

(Department of Internal Medicine and Cardiovascular Center, Seoul National University Hospital, Seoul (D.H., T.-M.R., J.P., B.-K.K., H.-S.K. ); Division of Cardiology, Department of Internal Medicine, Heart Vascular Stroke Institute, Samsung Medical Center, Sungkyunkwan University School of Medicine, Seoul (J.M.L., K.H.C., T.K.P., J.H.Y., Y.B.S., J.-H.C., J.-Y.H., S.-H.C., H.-C.G.); Department of Internal Medicine and

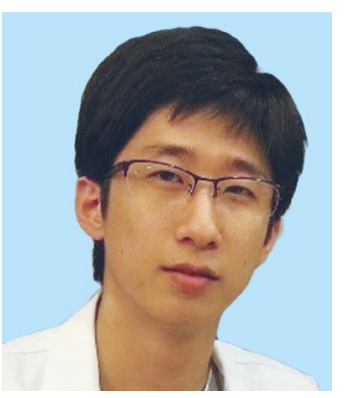
Cardiovascular Center, Chosun University Hospital, University of Chosun College of Medicine, Gwangju (H.K.K.); National Maritime Medical Center, Changwon (T.-M.R.); Department of Cardiology, Yeungnam University Medical Center, Daegu (Y.J.K.); School of Medicine, Kyungpook National University, Daegu (S.-C.C.); Cardiology Division, Department of Internal Medicine, Chungbuk National University Hospital, Cheongju (M. C. C. ); Department of Internal Medicine, Kyunghee University College of Medicine, Seoul (C.J.K.); and Department of Internal Medicine and Heart Center, Chonnam National University Hospital, Gwangju (M.H.J.), Korea)

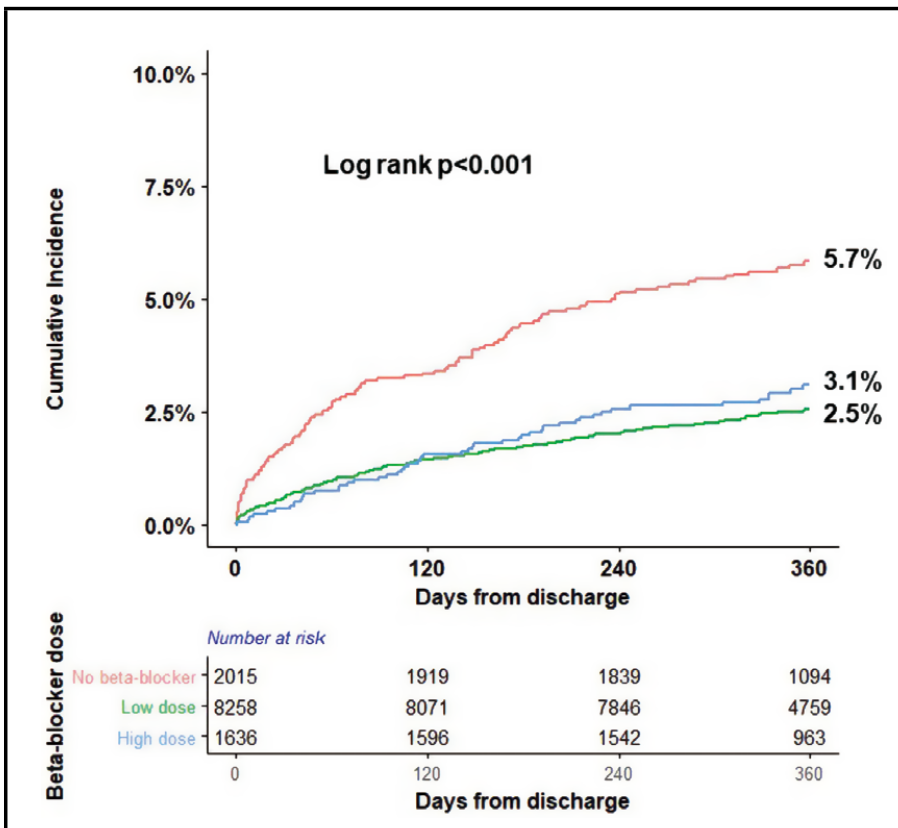

Figure 4. Cumulative incidence of cardiac death according to level of $\beta$-blocker dose at 1 year of $\beta$-blocker treatment.

Background: The differential prognostic impact of $\beta$-blocker dose after acute myocardial infarction (AMI) has been under debate. The current study sought to compare clinical outcome after AMI according to $\beta$-blocker dose using the Korea Acute Myocardial Infarction Registry-National Institutes of Health (KAMIR-NIH).

Methods and Results: Of the total population of 13,104 consecutive AMI patients enrolled in the KAMIR-NIH, the current study analyzed 11,909 patients. These patients were classified into 3 groups (no $\beta$-blocker; low-dose $[<25 \%$ of target dose]; and high-dose [ $\geq 25 \%$ of target dose]). The primary outcome was cardiac death at 1 year. Compared with the no $\beta$-blocker group, both the low-dose and high-dose groups had significantly lower risk of cardiac death (HR, $0.435 ; 95 \%$ CI: 0.363-0.521, P<0.001; HR, 0.519; 95\% CI: $0.350-0.772, \mathrm{P}=0.001$, respectively). The risk of cardiac death, however, was similar between the high- and low-dose groups (HR, 1.194; 95\% CI: $0.789-1.808, \mathrm{P}=0.402$ ). On multivariable adjustment and inverse probability weighted analysis, the result was the same.

Conclusions: The use of $\beta$-blockers in post-AMI patients had significant survival benefit compared with no use of $\beta$-blockers. There was no significant additional benefit of high-dose $\beta$-blockers compared with low-dose $\beta$-blockers, however, in terms of 1-year risk of cardiac death. 
Awards will be presented to the 7 research groups during the $84^{\text {th }}$ Annual Scientific Meeting of the Japanese Circulation Society, and will also be announced on the Society website. We look forward to receiving manuscripts with high scientific impact for publication in Circulation Journal in 2020.

\section{Toyoaki Murohara, MD, PhD \\ Editor-in-Chief \\ Circulation Journal}

\section{References:}

1. Shibata A, Mori H, Kodo K, Nakanishi T, Yamagishi H. Polysplenia syndrome as a risk factor for early progression of pulmonary hypertension. Circ J 2019; 83: 831-836.

2. Tsutsui H, Momomura S, Yamashina A, Shimokawa H, Kihara Y, Saito Y, et al; on behalf of the J-SHIFT Study Investigators. Efficacy and safety of ivabradine in Japanese patients with chronic heart failure: J-SHIFT Study. Circ J 2019; 83: $2049-2060$.

3. Yoshida S, Miyagawa S, Fukushima S, Yoshikawa Y, Hata H, Saito S, et al. Cardiac function and type of mitral valve surgery affect postoperative blood flow pattern in the left ventricle. Circ J 2019; 83: 130-138.

4. Kondo K, Miura K, Tanaka-Mizuno S, Kadota A, Arima H, Okuda N, et al; for the NIPPON DATA80 Research Group. Cardiovascular risk assessment chart by dietary factors in Japan: NIPPON DATA80. Circ J 2019; 83: 1254-1260.

5. Hayashi R, Iso H, Yamagishi K, Yatsuya H, Saito I, Kokubo Y, et al; for the Japan Public Health Center-Based (JPHC) Prospective Study Group. Working hours and risk of acute myocardial infarction and stroke among middle-aged Japanese men: The Japan Public Health Center-Based Prospective Study Cohort II. Circ J 2019; 83: 1072-1079.

6. Sato M, Miyata K, Tian Z, Kadomatsu T, Ujihara Y, Morinaga J, et al. Loss of endogenous HMGB2 promotes cardiac dysfunction and pressure overload-induced heart failure in mice. Circ $J$ 2019; 83: 368 -378.

7. Hwang D, Lee JM, Kim HK, Choi KH, Rhee TM, Park J, et al; The KAMIR Investigators. Prognostic impact of $\beta$-blocker dose after acute myocardial infarction. Circ J 2019; 83: 410-417. 